\title{
Additive spanners: A simple construction
}

\author{
Mathias Bæk Tejs Knudsen* \\ University of Copenhagen
}

November 25, 2014

\begin{abstract}
We consider additive spanners of unweighted undirected graphs. Let $G$ be a graph and $H$ a subgraph of $G$. The most naïve way to construct an additive $k$-spanner of $G$ is the following: As long as $H$ is not an additive $k$-spanner repeat: Find a pair $(u, v) \in H$ that violates the spanner-condition and a shortest path from $u$ to $v$ in $G$. Add the edges of this path to $H$.

We show that, with a very simple initial graph $H$, this naïve method gives additive 6 - and 2 -spanners of sizes matching the best known upper bounds. For additive 2 -spanners we start with $H=\varnothing$ and end with $O\left(n^{3 / 2}\right)$ edges in the spanner. For additive 6-spanners we start with $H$ containing $\left\lfloor n^{1 / 3}\right\rfloor$ arbitrary edges incident to each node and end with a spanner of size $O\left(n^{4 / 3}\right)$.
\end{abstract}

\section{Introduction}

Additive spanners are subgraphs that preserve the distances in the graph up to an additive positive constant. Given an unweighted undirected graph $G$, a subgraph $H$ is an additive $k$-spanner if for every pair of nodes $u, v$ it is true that

$$
d_{G}(u, v) \leqslant d_{H}(u, v) \leqslant d_{G}(u, v)+k
$$

In this paper we only consider purely additive spanners, which are $k$-spanners where $k=O(1)$. Throughout this paper every graph will be unweighted and undirected.

Many people have considered a variant of this problem, namely multiplicative spanners and even mixes between additive and multiplicative spanners [5, 4, 6]. The problem of finding a $k$-spanner of smallest size has received a lot of attention. Most notably, given a graph with $n$ nodes Dor et al. [3] prove that it has a 2-spanner of size $O\left(n^{3 / 2}\right)$, Baswana et al. [1] prove that it has a 6 -spanner of size $O\left(n^{4 / 3}\right)$, and Chechik [2] proves that it has a 4-spanner of size $O\left(n^{7 / 5} \log ^{1 / 5} n\right)$. Woodrufff [7] shows that for every constant $k$ there exist graphs with $n$ nodes such that every $(2 k-1)$-spanner must have at least $\Omega\left(n^{1+1 / k}\right)$ edges. This implies that the construction of 2 -spanners are optimal. Whether there exists an algorithm for constructing $O(1)$-spanners with $O\left(n^{1+\varepsilon}\right)$ edges for some $\varepsilon<1 / 3$ is unknown and is an important open problem.

Let $G$ be a graph and $H$ a subgraph of $G$. Consider the following algorithm: As long as there exists a pair of nodes $u, v$ such that $d_{H}(u, v)>d_{G}(u, v)+k$, find a shortest path from $u$ to $v$ in $G$ and add the edges on the path to $H$. This process will be referred to as $k$-spanner-completion. After $k$-spanner-completion, $H$ will be a $k$-spanner of $G$. Thus, given a graph $G$, a general way to construct a $k$-spanner for $G$ is the following: Firstly, find a simple subgraph of $G$. Secondly use $k$-spanner-completion on this subgraph. The main contribution of this paper is:

Theorem 1.1. Let $G$ be a graph with $n$ nodes and $H$ the subgraph containing all nodes but no edges of $G$. For each node add $\left\lfloor n^{1 / 3}\right\rfloor$ edges adjacent to that node to $H$ (or, if the degree is less, add all edges incident to that node). After 6-spanner-completion $H$ will have at most $O\left(n^{4 / 3}\right)$ edges.

It is well-known that a graph with $n$ nodes has a 6 -spanner of size $O\left(n^{4 / 3}\right)[1$. The techniques employed in our proof of correctness are similar to those in [1. The creation of the initial graph $H$ corresponds to the clustering in [1] and the 6-spanner-completion corresponds to their path-buying algorithm. For completeness we show that the same method gives a 2-spanner of size $O\left(n^{3 / 2}\right)$. This fact is already known due to [3] and is matched by a lower bound from 7 .

\footnotetext{
* Research partly supported by Thorup's Advanced Grant from the Danish Council for Independent Research under the Sapere Aude research carrier programme and by the FNU project AlgoDisc - Discrete Mathematics, Algorithms, and Data Structrues
} 
Theorem 1.2. Let $G$ be a graph with $n$ nodes and $H$ the subgraph where all edges are removed. Upon 2-spanner-completion $H$ has at most $O\left(n^{3 / 2}\right)$ edges.

\section{Creating a 6-spanner}

The algorithm for creating a 6 -spanner was described in the abstract and the introduction.

For a given graph $G$, a 6 -spanner of $G$ can be created by strating with some subgraph $H$ of $G$ and applying 6-spanner-completion to $H$. Theorem 1.1 states that for a suitable starting choice of $H$ we get a spanner of size $O\left(n^{4 / 3}\right)$. The purpose of this section is to show that the 6 -spanner created has no more than $O\left(n^{4 / 3}\right)$ edges. This will imply that the construction (in terms of the size of the 6 -spanner) matches the best known upper bound [1].

of Theorem 1.1. Inserting (at most) $\left\lfloor n^{1 / 3}\right\rfloor$ edges per node will only add $n\left\lfloor n^{1 / 3}\right\rfloor=O\left(n^{4 / 3}\right)$ edges to $H$. Therefore it is only necessary to prove that 6 -spanner-completion adds no more than $O\left(n^{4 / 3}\right)$ edges.

Let $v(H)$ and $c(H)$ be defined by:

$$
v(H)=\sum_{u, v \in V(G)} \max \left\{0, d_{G}(u, v)-d_{H}(u, v)+5\right\}, \quad c(H)=\# E(H)
$$

Say that a shortest path, $p$, from $u$ to $v$ is added to $H$, and let $H_{0}$ be the subgraph before the edges are added. Let the path consist of the nodes:

$$
u=w_{0}, w_{1}, \ldots, w_{r}=v, r \in \mathbb{N}
$$

Let $u^{\prime}=w_{i}$ be the node $w_{i}$ with the smallest $i$ such that $\operatorname{deg}_{H_{0}}\left(w_{i}\right) \geqslant\left\lfloor n^{1 / 3}\right\rfloor$. Likewise let $v^{\prime}=w_{j}$ be the node $w_{j}$ the largest $j$ such that $\operatorname{deg}_{H_{0}}\left(w_{j}\right) \geqslant\left\lfloor n^{1 / 3}\right\rfloor$. Remember that if $\operatorname{deg}_{H_{0}}\left(w_{i}\right)<\left\lfloor n^{1 / 3}\right\rfloor$ then all the edges adjacent to $w_{i}$ are already in $H_{0}$. This implies that $d_{H_{0}}\left(u^{\prime}, v^{\prime}\right)>d_{G}\left(u^{\prime}, v^{\prime}\right)+6$ since $d_{H_{0}}(u, v)>d_{G}(u, v)+6$.

Say that $t$ new edges are added to $H$. Then there must be at least $t$ nodes on $p$ with degree $>n^{1 / 3}$. Since every node can be adjacent to no more than 3 nodes on $p$ (since it is a shortest path) there must be $\Omega\left(n^{1 / 3} t\right)$ nodes adjacent to $p$ in $H$. Let $z$ and $w$ be neighbours to $u^{\prime}$ and $v^{\prime}$ in $H$ respectively and let $r$ be any node adjacent to $p$ in $H$. Let $s$ be a node on $p$ such that $r$ and $s$ are adjacent in $H$. See Figure 1 for an illustration.

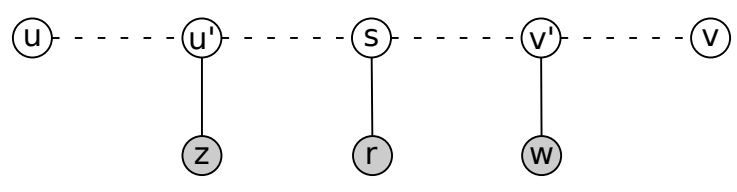

Figure 1: The dashed line denotes the shortest path from $u$ to $v$. The solid lines denote edges.

By the triangle inequality we see that:

$$
d_{H}(z, r)+d_{H}(r, w) \leqslant d_{G}\left(u^{\prime}, v^{\prime}\right)+4
$$

But on the other hand:

$$
d_{H_{0}}(z, r)+d_{H_{0}}(r, w) \geqslant d_{H_{0}}(z, w) \geqslant d_{H_{0}}\left(u^{\prime}, v^{\prime}\right)-2>d_{G}\left(u^{\prime}, v^{\prime}\right)+4
$$

Combining these two inequalities we obtain $d_{H_{0}}(z, r)>d_{H}(z, r)$ or $d_{H_{0}}(r, w)>d_{H}(r, w)$. And from the triangle inequality $d_{G}(z, r)+5>d_{H}(z, r)$ and $d_{G}(r, w)+5>d_{H}(r, w)$. Since $u^{\prime}$ and $v^{\prime}$ have at least $n^{1 / 3}$ neighbours and there are $\Omega\left(n^{1 / 3} t\right)$ nodes in $H$ adjacent to $p$, the definition of $v(H)$ implies that:

$$
v(H)-v\left(H_{0}\right) \geqslant \Omega\left(t\left(n^{1 / 3}\right)^{2}\right)
$$

And since $c(H)-c\left(H_{0}\right)=t$ :

$$
\frac{v(H)-v\left(H_{0}\right)}{c(H)-c\left(H_{0}\right)} \geqslant \Omega\left(n^{2 / 3}\right)
$$

Since $v(H) \leqslant O\left(n^{2}\right)$ this implies that $c(H)$ increases with no more than $O\left(n^{2} / n^{2 / 3}\right)=O\left(n^{4 / 3}\right)$ in total when all shortest paths are inserted. Hence $c(H)=O\left(n^{4 / 3}\right)$ when the 6 -spanner-completion is finished which yields the conclusion. 


\section{Creating a 2-spanner}

For completeness we show that 2-spanner-completion gives spanners with $O\left(n^{3 / 2}\right)$ edges. This matches the upper bound from [3] and the lower bound from [7].

of Theorem 1.2. Let $G$ be a graph with $n$ nodes. Whenever $H$ is a spanner of $G$, define $v(H)$ and $c(H)$ as:

$$
v(H)=\sum_{u, v \in V(G)} \max \left\{0, d_{G}(u, v)-d_{H}(u, v)+3\right\}, \quad c(H)=\sum_{v \in V(G)}\left(\operatorname{deg}_{H}(v)\right)^{2}
$$

It is easy to see that $0 \leqslant v(H) \leqslant 3 n^{2}$ and by Cauchy-Schwartz's inequality $\sqrt{c(H) \cdot n} \geqslant 2 \# E(H)$. The goal will be to prove that when the algorithm terminates $c(H)=O\left(n^{2}\right)$, since this implies that $\# E(H)=O\left(n^{3 / 2}\right)$. This is done by proving that in each step of the algorithm $c(H)-12 v(H)$ will not increase. Since $v(H)=O\left(n^{2}\right)$ this means that $c(H)=O\left(n^{2}\right)$ which ends the proof. Therefore it is sufficient to check that $c(H)-12 v(H)$ never increases.

Consider a step where new edges are added to $H$ on a shortest path from $u$ to $v$ of length $t$. Let $H_{0}$ be the subgraph before the edges are added. Assume that $u, v$ violates the 2 -spanner condition in $H_{0}$, i.e. $d_{H_{0}}(u, v)>d_{G}(u, v)+2$. Let the shortest path consist of the nodes:

$$
u=w_{0}, w_{1}, \ldots, w_{t-1}, w_{t}=v
$$

It is obvious that:

$$
c(H)-c\left(H_{0}\right) \leqslant \sum_{i=0}^{t}\left(\operatorname{deg}_{H}\left(w_{i}\right)\right)^{2}-\left(\operatorname{deg}_{H}\left(w_{i}\right)-2\right)^{2} \leqslant 4 \sum_{i=0}^{t} \operatorname{deg}_{H}\left(w_{i}\right)
$$

Every node cannot be adjacent to more than 3 nodes on the shortest path, since otherwise it would not be a shortest path. Using this insight we can bound the number of nodes which in $H$ are adjacent to or on the shortest path from below by:

$$
\frac{1}{3} \sum_{i=0}^{t} \operatorname{deg}_{H}\left(w_{i}\right)
$$

Now let $z$ be a node in $H$ adjacent or on to the shortest path. Obviously:

$$
d_{H}(u, z)+d_{H}(z, v) \leqslant d_{G}(u, v)+2
$$

Furthermore $d_{H_{0}}(u, z)+d_{H_{0}}(z, v)>d_{G}(u, v)+2$ since otherwise there would exist a path from $u$ to $v$ in $H_{0}$ of length $\leqslant d_{G}(u, v)+2$. Hence:

$$
d_{H}(u, z)+d_{H}(z, v)<d_{H_{0}}(u, z)+d_{H_{0}}(z, v)
$$

Now let $z$ be a node on the shortest path which is adjacent to $w_{i}$ in $H$ (every node on the path will also be adjacent in $H$ to such a node). Then by the triangle inequality:

$$
\begin{aligned}
d_{H}(u, z) & \leqslant d_{H}\left(u, w_{i}\right)+d_{H}\left(w_{i}, z\right)=d_{G}\left(u, w_{i}\right)+1 \\
& \leqslant d_{G}(u, z)+d_{G}\left(z, w_{i}\right)+1=d_{G}(u, z)+2
\end{aligned}
$$

And likewise $d_{H}(z, v) \leqslant d_{G}(z, v)+2$. Combining these two observations yields:

$$
\sum_{w \in V} \max \left\{0, d_{G}(z, w)-d_{H}(z, w)+3\right\}<\sum_{w \in V} \max \left\{0, d_{G}(z, w)-d_{H_{0}}(z, w)+3\right\}
$$

Since this holds for every node in $H$ adjacent to or on the shortest path this means that:

$$
v(H)-v\left(H_{0}\right) \geqslant \frac{1}{3} \sum_{i=0}^{t} \operatorname{deg}_{H}\left(w_{i}\right)
$$

Combining this with the bound on $c(H)-c\left(H_{0}\right)$ gives:

$$
(c(H)-12 v(H))-\left(c\left(H_{0}\right)-12 v\left(H_{0}\right)\right) \leqslant 0
$$

which finishes the proof. 


\section{References}

[1] Surender Baswana, Telikepalli Kavitha, Kurt Mehlhorn, and Seth Pettie. New constructions of $(\alpha, \beta)$-spanners and purely additive spanners. In Proc. 16th ACM/SIAM Symposium on Discrete Algorithms (SODA), pages 672-681, 2005.

[2] Shiri Chechik. New additive spanners. In Proc. 24th ACM/SIAM Symposium on Discrete Algorithms (SODA), pages 498-512, 2013.

[3] Dorit Dor, Shay Halperin, and Uri Zwick. All-pairs almost shortest paths. SIAM Journal on Computing, 29(5):1740-1759, 2000. See also FOCS'96.

[4] Michael Elkin and David Peleg. $(1+\varepsilon, \beta)$-spanner constructions for general graphs. SIAM Journal on Computing, 33(3):608-631, 2004. See also STOC'01.

[5] Seth Pettie. Low distortion spanners. In Proc. 34th International Colloquium on Automata, Languages and Programming (ICALP), pages 78-89, 2007.

[6] Mikkel Thorup and Uri Zwick. Spanners and emulators with sublinear distance errors. In Proc. 17th ACM/SIAM Symposium on Discrete Algorithms (SODA), pages 802-809, 2006.

[7] David P. Woodruff. Lower bounds for additive spanners, emulators, and more. In Proc. 47 th IEEE Symposium on Foundations of Computer Science (FOCS), pages 389-398, 2006. 